\title{
Hair follicles' transit-amplifying cells govern concurrent dermal adipocyte production through Sonic Hedgehog
}

\author{
Bing Zhang, 1,2,6 Pai-Chi Tsai, ${ }^{1,2,6}$ Meryem Gonzalez-Celeiro, ${ }^{1,2,3}$ Oliver Chung, ${ }^{1,2}$ \\ Benjamin Boumard, ${ }^{1,2}$ Carolina N. Perdigoto, ${ }^{4,5}$ Elena Ezhkova, ${ }^{4,5}$ and Ya-Chieh Hsu ${ }^{1,2}$ \\ ${ }^{1}$ Department of Stem Cell and Regenerative Biology, Cambridge, Massachusetts 02138, USA; ${ }^{2}$ Harvard Stem Cell Institute, \\ Harvard University, Cambridge, Massachusetts 02138, USA; ${ }^{3}$ Institute of Molecular Health Sciences, Eidgenössische Technische \\ Hochschule Zurich, 8093 Zurich, Switzerland; ${ }^{4}$ Black Family Stem Cell Institute, ${ }^{5}$ Department of Developmental and \\ Regenerative Biology, Icahn School of Medicine at Mount Sinai, New York, New York 10029, USA
}

\begin{abstract}
Growth and regeneration of one tissue within an organ compels accommodative changes in the surrounding tissues. However, the molecular nature and operating logic governing these concurrent changes remain poorly defined. The dermal adipose layer expands concomitantly with hair follicle downgrowth, providing a paradigm for studying coordinated changes of surrounding lineages with a regenerating tissue. Here, we discover that hair follicle transitamplifying cells (HF-TACs) play an essential role in orchestrating dermal adipogenesis through secreting Sonic Hedgehog (SHH). Depletion of Shh from HF-TACs abrogates both dermal adipogenesis and hair follicle growth. Using cell type-specific deletion of Smo, a gene required in SHH-receiving cells, we found that SHH does not act on hair follicles, adipocytes, endothelial cells, and hematopoietic cells for adipogenesis. Instead, SHH acts directly on adipocyte precursors, promoting their proliferation and their expression of a key adipogenic gene, peroxisome proliferator-activated receptor $\gamma$ (Pparg), to induce dermal adipogenesis. Our study therefore uncovers a critical role for TACs in orchestrating the generation of both their own progeny and a neighboring lineage to achieve concomitant tissue production across lineages.
\end{abstract}

[Keywords: transit-amplifying cells; adipogenesis; interlineage communications; hair follicle regeneration; adipocyte precursors; stroma]

Supplemental material is available for this article.

Received June 12, 2016; revised version accepted October 3, 2016.

Mammalian organs are functional units composed of cells from diverse lineages. Current studies often focus on how individual lineages grow and regenerate. However, mass production of one lineage can impose challenges on the organ in which it resides. The neighboring tissues must undergo concurrent and often substantial remodeling to create new space and structural and signaling support for this newly generated tissue. How coordinated changes across diverse lineages within a complex mammalian organ are orchestrated is an important question that remains to be resolved.

The skin provides an ideal model to understand how changes of the surrounding niche can occur concurrently to accommodate a rapidly growing tissue. The skin harbors a rich array of cell types, including epidermis, hair follicles, dermal fibroblasts, and adipocytes (Blanpain and Fuchs 2009; Hsu et al. 2014a; Chen et al. 2016; Shook

${ }^{6}$ These authors contributed equally to this work.

Corresponding author: yachieh_hsu@harvard.edu

Article published online ahead of print. Article and publication date are online at http://www.genesdev.org/cgi/doi/10.1101/gad.285429.116. et al. 2016; Xin et al. 2016). Among them, hair follicles undergo cyclical rounds of growth (anagen), regression (catagen), and rest (telogen). Anagen is initiated by hair follicle stem cells in the hair germ and bulge. However, hair follicle downgrowth is driven mostly by the fastcycling transit-amplifying cells of the hair follicle (HF-TACs), a population generated by the stem cells to produce large amounts of downstream progeny (Hsu et al. 2014b). During catagen, HF-TACs are destroyed, and the hair follicles are remolded back to their telogen morphology.

The hair follicles are one of the most rapidly growing tissues in adults: Within $7 \mathrm{~d}$, hair follicles grow from rudimentary hair germs to complex full-anagen hair follicles that are 60 times longer (Supplemental Fig. S1A). Rapid downgrowth of hair follicles compels the surrounding

(C) 2016 Zhang et al. This article is distributed exclusively by Cold Spring Harbor Laboratory Press for the first six months after the full-issue publication date (see http://genesdev.cshlp.org/site/misc/terms.xhtml). After six months, it is available under a Creative Commons License (Attribution-NonCommercial 4.0 International), as described at http://creativecommons.org/licenses/by-nc/4.0/. 
dermis to expand concomitantly in order to avoid hair follicles abutting against the panniculus carnosus, a layer of striated muscle underneath the dermal adipocytes (Fig. 1A). This dermal expansion is facilitated by the thickening of the dermal adipose layer during anagen, which results in a drastically thicker anagen skin compared with the telogen skin (Festa et al. 2011; Donati et al. 2014). Dermal adipocytes and adipocyte precursors influence hair cycle (Plikus et al. 2008; Festa et al. 2011; Keyes et al. 2013). In addition, dermal adipocytes secrete antimicrobial peptides to protect the skin from bacterial infection, thereby playing a role in the skin's innate immunity (Zhang et al. 2015). Ectopic activation of $\beta$-catenin in the epithelial compartment affects several aspects of skin biology, including hair cycle, dermal fibroblasts, and adipose layer thickness (Deschene et al. 2014; Donati et al. 2014; Kretzschmar et al. 2016; Lichtenberger et al. 2016), suggesting that dermal changes are sensitive to alterations in the epithelial lineage. However, the physio- logically relevant factor and the specific cell types governing the coordinated changes of dermal adipose layer and hair cycle progression remain unknown.

Here, we identify mechanisms by which hair cycles and dermal adipogenesis are coupled. We demonstrate that new dermal adipocytes begin to emerge right after HFTAC formation in mid-anagen, continue throughout anagen, and cease at catagen after HF-TACs are destroyed. This tight association of HF-TACs and dermal adipogenesis is due to Sonic Hedgehog (SHH) secreted from HFTACs. Through cell type-specific manipulations, we show that $\mathrm{SHH}$ promotes adipocyte precursor proliferation and regulates the expression of peroxisome proliferator-activated receptor $\gamma$ (Pparg), a key adipogenesis gene. Our study therefore uncovers a mechanism by which dermal adipogenesis couples with hair follicle regeneration and points to an unanticipated role of TACs in orchestrating concurrent production of a neighboring tissue in addition to their own progeny.

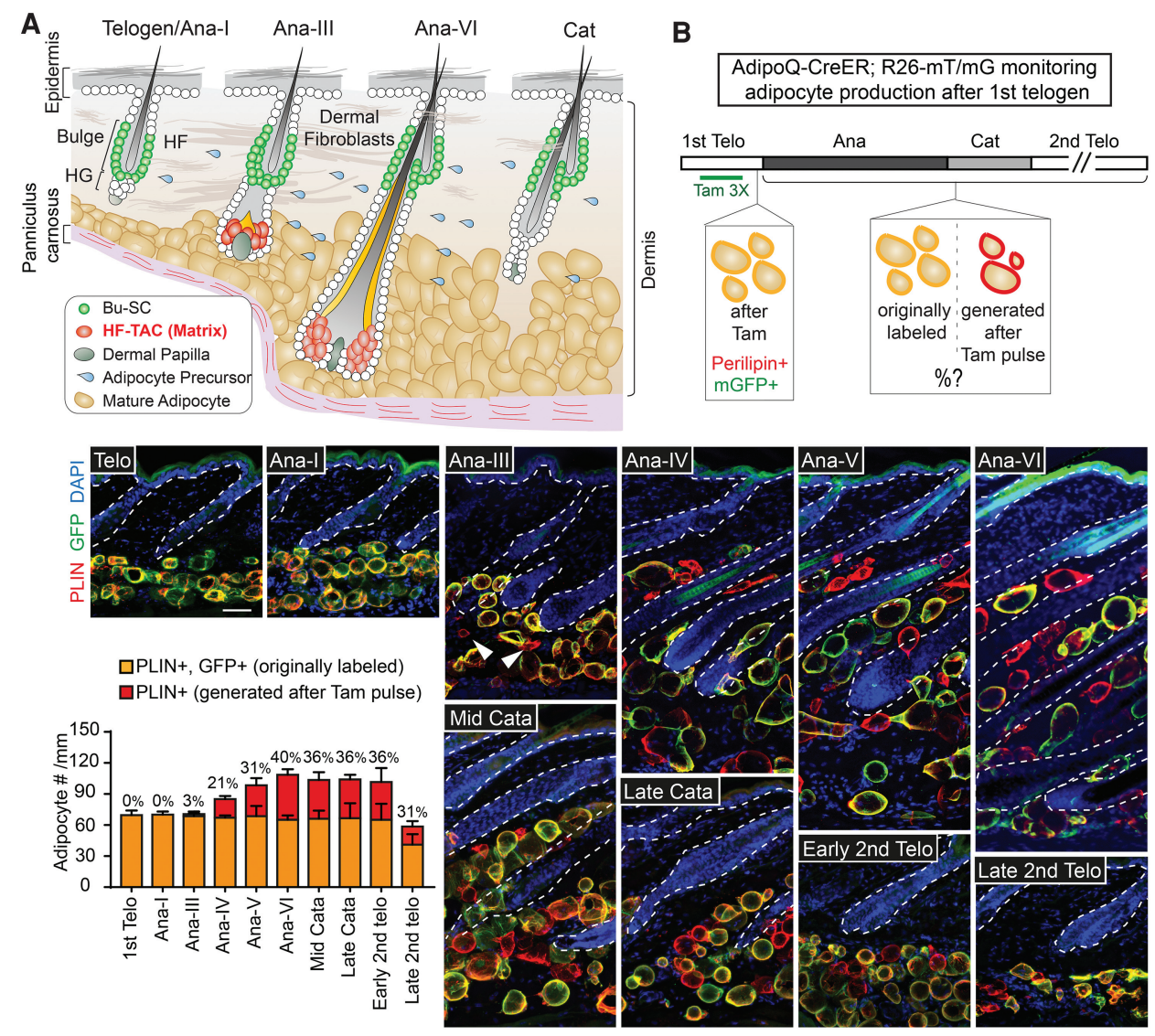

Figure 1. Dermal adipogenesis commences after the formation of HF-TACs and stops upon HF-TAC destruction. $(A)$ Schematic of the skin at distinct hair cycle stages illustrating different cell types and their relative positions. HF-TACs are formed at mid-anagen and are absent in telogen, anagen I (Ana-I), or catagen hair follicles. (B) Tamoxifen (Tam)-treated AdipoQ-CreER; Rosa26-lox membrane tdTomato-lox membrane GFP $(R 26-\mathrm{mT} / \mathrm{mG})$ mice taken at different hair cycle stages. Tamoxifen treatment (three times, $3 \times)$ leads to activation of membrane GFP (mGFP) in existing adipoctyes at the first telogen, which appears as membrane GFP- and Perilipin (PLIN)-doublepositive (yellow). Adipocytes generated after this labeling period are Perilipin-positive but negative for membrane GFP (red). The bar graph quantifies the number of adipocytes found in each millimeter width of skin. The percentages on the bar graph denote the percentage of adipocytes generated after initial tamoxifen labeling among total adipocytes found at each hair cycle substage. (Arrowheads) Newly generated adipocytes. $n=2$ mice for each stage. Data are mean \pm SD. Bars, $50 \mu \mathrm{m}$. 


\section{Results}

New dermal adipocytes begin to form at mid-anagen and cease at catagen

The full-anagen skin is substantially thicker than the telogen skin, with longer hair follicles and a thickened adipose layer. To reach this state, the dermis might undergo morphological changes at anagen onset in preparation for the expected hair follicle regeneration. Alternatively, regenerating hair follicles may actively instruct the surrounding dermis to gradually expand together. To distinguish between these possibilities, we first examined exactly when dermal thickness begins to change. Anagen can be divided into six substages (Muller-Rover et al. 2001). Prominent morphological changes occur in the hair follicles as soon as anagen begins: The hair germ proliferates and enlarges at anagen I (Ana-I), and stem cells in the bulge (Bu-SCs) are activated at Ana-II. This proliferation leads to generation of HF-TACs, also known as the matrix, at the transition from Ana-II to Ana-III /Greco et al. 2009; Hsu et al. 2014b). In contrast, we found that the dermis does not display noticeable changes until Ana-III, when the HF-TACs form. From Ana-III onward, the dermis expands proportionally to the downgrowing hair follicles. The increase in dermal thickness is mediated primarily through the expansion of the dermal adipose layer, not the fibroblast layer (Supplemental Fig. S1B).

To determine the timing when new adipocytes start to emerge, we used a lineage-tracing strategy combining AdipoQ-CreER, an inducible Cre expressed in mature adipocytes (Jeffery et al. 2014), together with R26-mT/mG mice, which express membrane tdTomato $(\mathrm{mT})$ before Cre excision and membrane GFP (mG) after Cre activation (Muzumdar et al. 2007). Since membrane tdTomato is ubiquitously expressed, we relied on Perilipin (which marks the surface of lipid droplets) staining instead of tdTomato to visualize all adipocytes. AdipoQ-CreER was inactive without tamoxifen (Supplemental Fig. S2A). We tested the efficacy of AdipoQ-CreER and found that with three doses of tamoxifen at telogen, virtually all dermal adipocytes were effectively marked with membrane GFP and appeared as GFP- and Perilipin-double-positive (Fig. 1B, shown in yellow; Supplemental Fig. S2B). Adipocytes that emerged after this labeling period became GFP-negative but Perilipin-positive (Fig. 1B, shown in red). While dermal adipocytes remained as GFP- and Perilipin-double-positive before Ana-II, new adipocytes began to emerge sparsely at Ana-III (Fig. 1B, arrowheads). The number of new adipocytes increased with anagen progression (Fig. 1B, red bars). In contrast, the number of GFP-labeled adipocytes remained constant throughout anagen (Fig. 1B, yellow bars), suggesting that adipocytes from telogen do not undergo significant turnover during the subsequent anagen.

HF-TACs are present only in anagen. To determine whether dermal adipogenesis is tightly linked with HFTACs, we followed the marked AdipoQ-CreER; R26$\mathrm{mT} / \mathrm{mG}$ skin into catagen, when HF-TACs degenerate, and the following second telogen. Adipogenesis ceased right after HF-TACs were destroyed in catagen (Fig. 1B).
Interestingly, the total number of adipocytes as well as the ratio of originally labeled adipocytes versus adipocytes generated after tamoxifen pulse remained constant at Ana-VI (the last stage of anagen), catagen, and the beginning of the second telogen. Adipocyte numbers were reduced by late second telogen (Fig. 1B). These data suggest that adipogenesis stops right after TAC destruction in catagen. Moreover, most of the adipocytes were lost during the prolonged second telogen stage rather than in catagen. A complementary lineage tracing by treating AdipoQ-CreER; R26- $\mathrm{mT} / \mathrm{mG}$ mice with tamoxifen at Ana-VI and examining labeling results at Ana-VI versus the beginning of the second telogen also confirmed that few if any adipocytes were produced during catagen and telogen (Supplemental Fig. S2C). Together, these data suggest that once HF-TACs are destroyed, adipocyte production stops concomitantly.

To demonstrate unequivocally that anagen entry precedes dermal adipogenesis, we looked into dermal changes after hair depilation (plucking) (Hsu et al. 2011). Depilation allows anagen to begin at any chosen postnatal day during telogen. We first labeled all pre-existing adipocytes during the extended second telogen in AdipoQCreER; R26-mT/mG mice with tamoxifen followed by hair plucking. New adipocytes were consistently observed in the plucked area $6 \mathrm{~d}$ after plucking (Supplemental Fig. S2D, arrowhead), when the hair follicles entered Ana-III. However, no new adipocytes were found $2 \mathrm{~d}$ after plucking, when hair follicles were at Ana-I. Dermal adipogenesis occurred specifically underneath the plucked region. The hair follicles right next to the depilated spot remained in telogen, and adipogenesis was not observed underneath these telogen hair follicles (Supplemental Fig. S2D). Collectively, these data suggest that, instead of occurring at a defined postnatal day, dermal adipogenesis begins concomitantly with HF-TAC formation and ceases when HF-TACs are destroyed.

\section{TAC-derived SHH is required for dermal adipogenesis}

The tight coupling of HF-TAC emergence and adipogenesis raises the interesting possibility that signals derived from HF-TACs might orchestrate dermal adipogenesis. SHH is a known factor secreted by HF-TACs to promote hair follicle downgrowth and is not detected in telogen, catagen, or the beginning of anagen. SHH is also not detected in other cells of epidermal or dermal origins (Hsu et al. 2014b). The timing of Shh up-regulation in the skin makes it an attractive candidate to investigate.

To test whether TAC-derived SHH is involved in dermal adipogenesis, we knocked out $\mathrm{Shh}$ from hair follicles using K15-CrePGR, which is expressed in the Bu-SCs and hair germ that gives rise to HF-TACs (Ito et al. 2005). Knocking out Shh with K15-CrePGR affects the proliferation of both Bu-SCs and HF-TACs. The hair follicles can enter anagen but are arrested at Ana-II (Hsu et al. 2014b). Interestingly, the dermal adipose layer in K15CrePGR; Shhfl/fl mice failed to expand even $7 \mathrm{~d}$ after 
A
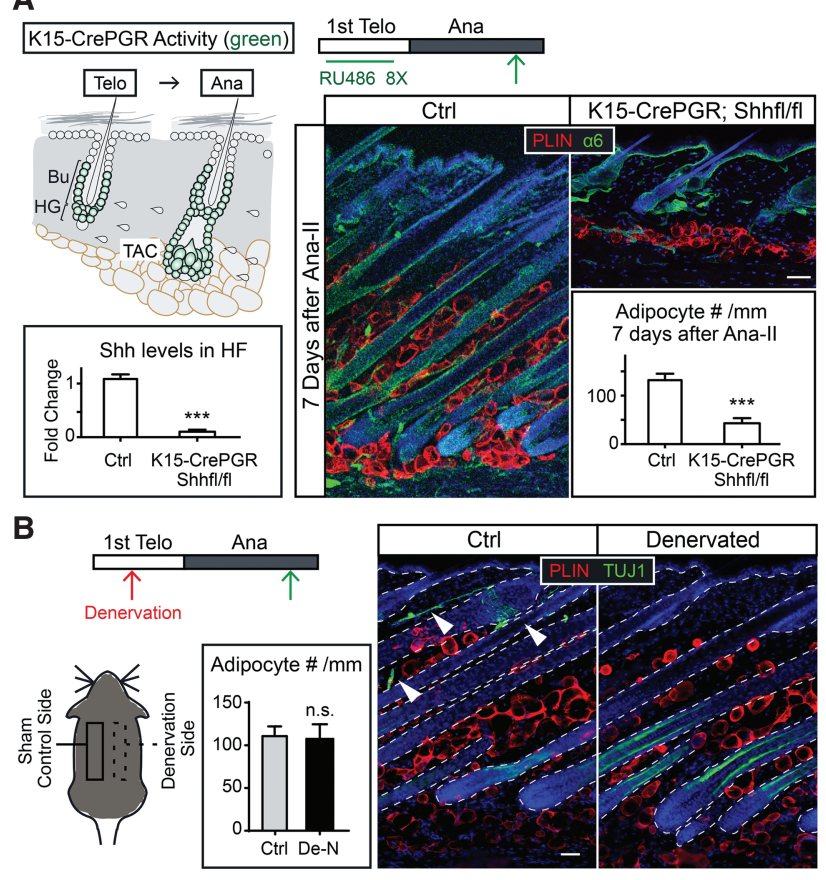

Figure 2. Depletion of Shh from HF-TACs abrogates anagen-associated dermal adipogenesis, while nerve-derived SHH is dispensable. (A) Conditional deletion of $\mathrm{Shh}$ from the adult hair follicles (using K15-CrePGR) prior to anagen entry inhibits both hair follicle downgrowth and dermal adipogenesis. Schematics show a K15-CrePGR induction scheme and cells that are positive for Cre activity (denoted in green) after K15-CrePGR is induced in telogen by RU486. Green arrows denote the time points when skin samples were taken. Integrin a 6 staining marks the basement membrane separating epithelial cells and dermal cells. Shh levels in the hair follicles (HF) were determined by RTPCR of FACS-purified $\mathrm{YFP}^{+}$cells isolated from K15-CrePGR; R26-1sl-YFP and K15-CrePGR; R26-1sl-YFP; Shhfl/f1 skin. Adipocyte numbers are quantified as numbers of Perilipin ${ }^{+}\left(\mathrm{PLIN}^{+}\right)$cells per millimeter of skin width. $n=2$ mice for control; $n=4$ mice for knockout. (B) Immunolabeling of Tuj1 (a pan-neuronal marker that also marks inner hair follicles) and Perilipin on the denervated (De-N) side and the sham-operated control side of the same mouse. Denervation was conducted in telogen, and the skin samples were taken in full anagen. $n=3$ mice. (Arrowheads) Nerve fibers. Data are mean \pm SD. Bars, $50 \mu \mathrm{m}$. (***) $P<0.001$; (n.s.) not significant.

Ana-II, suggesting that HF-TAC-derived SHH not only influences hair follicle downgrowth but also affects dermal adipogenesis (Fig. 2A).

Shh is also expressed in the sensory nerves innervating the hair follicles (Brownell et al. 2011). The nerve-derived $\mathrm{SHH}$ is dispensable for hair follicle growth, and its expression is at least 20 times lower than the HF-TAC-derived $\mathrm{SHH}$ (Hsu et al. 2014b). To test whether nerve-derived $\mathrm{SHH}$ plays a role in dermal adipogenesis, we performed denervation experiments. A prominent increase in adipocyte numbers was observed in both the denervated side and the sham-operated side when hair follicles reached full anagen, suggesting that nerve-derived $\mathrm{SHH}$ is dispensable for dermal adipogenesis (Fig. 2B).
SHH signaling is not required in the hair follicles for the expansion of the dermal adipose layer

Our data thus far suggest that dermal adipogenesis is impaired when Shh is depleted from HF-TACs. Because dermal adipogensis does not normally commence until midanagen, the lack of adipogenesis in Shh mutants might be due to the arrest of their hair follicle growth at early anagen. Alternatively, $\mathrm{SHH}$ may act on a specific cell type in the skin to influence dermal adipogenesis. To distinguish between these models, we investigated the requirement of Smoothened (Smo), an obligatory component of the SHH pathway in the signal-receiving cells, in different cell populations of the skin.

We first examined whether Smo is required in the hair follicles for adipogenesis by knocking out Smo with K15-CrePGR in telogen and followed the skin into anagen. Adult hair follicles lacking Smo can initiate anagen and grow downward despite being shorter at late anagen (Hsu et al. 2014b). The dermal adipocyte numbers were not significantly altered in K15-CrePGR; Smofl/fl skin at late anagen despite significant reduction in $S m o$ and SHH's downstream target, Gli1, in the hair follicles (Fig. $3 \mathrm{~A}-\mathrm{C})$. These data suggest that Smo activity in the hair follicle is not essential for adipogenesis. Moreover, these data rule out the possibility that $\mathrm{SHH}$ might elicit a secondary signal from the hair follicles to instruct dermal adipogenesis.

\section{SHH pathway activity is not required in the mature adipocyte for dermal adipogenesis}

We next examined whether $\mathrm{SHH}$ signaling is required by mature dermal adipocytes. For this, we turned to AdipoQ-CreER; Smofl/f1 mice. We induced AdipoQ-CreER at telogen and continued throughout anagen if the samples were taken at anagen to ensure that all dermal adipocytes were devoid of Smo (Fig. 3D). The knockout efficiency of Smo and reduction of Gli1 in mature adipocytes were also confirmed by RT-PCR in isolated dermal adipocytes (Fig. 3E,F). Despite efficient knockout, we observed no significant differences in adipocyte numbers in AdipoQ-CreER; Smofl/f1 skin in either telogen or anagen (Fig. 3D; Supplemental Fig. S3). These data suggest that $\mathrm{SHH}$ pathway activity is not required in mature adipocytes for dermal adipogenesis. Moreover, the SHH pathway is dispensable in mature adipocytes for maintaining adipose layer thickness.

\section{Identification of inducible Cre lines that mark distinct subsets of dermal fibroblasts}

Previous studies showed that mature adipocytes are derived from adipocyte precursors, which are a specialized subset of dermal fibroblasts that are $\mathrm{Pdgfra}^{+}, \mathrm{Sca}^{+}$, $\mathrm{CD}_{4}{ }^{+}, \mathrm{CD}^{-} 5^{-}$, and CD31- (Festa et al. 2011; Donati et al. 2014). To examine whether SHH signaling might be directly required by these adipocyte precursors, we first identified inducible Cre lines that are active in distinct dermal fibroblast populations. We began by 


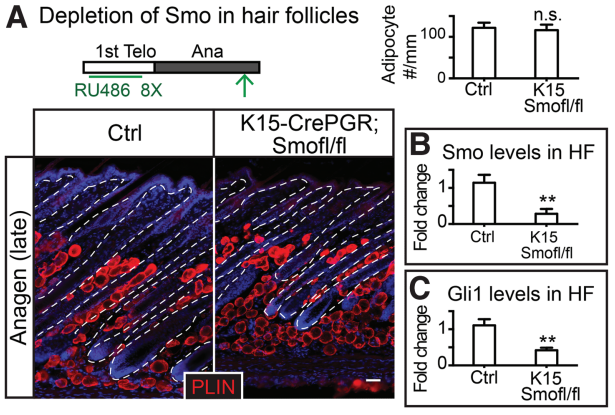

D Depletion of Smo in mature adipocytes

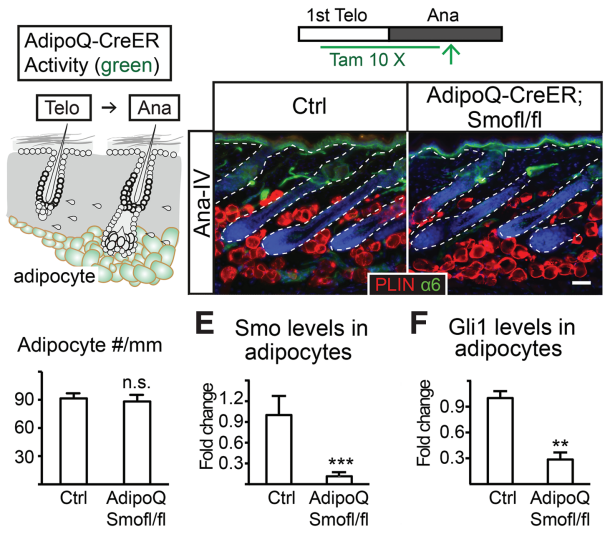

Figure 3. SHH pathway activity is not required in the hair follicles or mature adipocytes to promote adipogenesis in anagen. (A) Depletion of Smo with K15-CrePGR results in no changes in dermal adipocyte numbers. Bars represent the number of Perilipin $^{+}$cells per millimeter width of skin in each genotype. $n=2$ for control; $n=3$ mice for knockout. $(B, C)$ RT-PCR of Smo $(B)$ and Gli1 $(C)$ in hair follicles isolated from control and K15CrePGR; Smof/f1 anagen skin. (D) Deleting Smo with AdipoQCreER throughout telogen and anagen results in no change in dermal adipocyte numbers. The schematic represents an induction scheme and cells positive for Cre activity after 10 tamoxifen treatments $(10 x)$ throughout telogen and anagen. The bar graph quantifies the number of Perilipin ${ }^{+}$cells per millimeter width of skin. $(E, F)$ RT-PCR of Smo $(E)$ and Gli1 $(F)$ in mature dermal adipocytes isolated from control and AdipoQ-CreER; $S m o f / f 1$ anagen skin. $n=4$ mice for each genotype. The epidermis and hair follicles are outlined by white dashed lines. Data are mean \pm SD. Bars, $50 \mu \mathrm{m}$. $\left(^{* * *}\right) P<0.001$; $\left(^{* *}\right) P<0.01$; (n.s.) not significant.

focusing on Pdgfra-CreER (Kang et al. 2010). When induced in telogen, Pdgfra-CreER marked $>70 \%$ of the adipocyte precursors and other fibroblasts $\left(\mathrm{Pdgfra}^{+}, \mathrm{CD} 45^{-}\right.$, and $\left.\mathrm{CD}^{-}\right)$but not mature dermal adipocytes in telogen. In addition, hair follicles, endothelial cells, and immune cells were devoid of Pdgfra-CreER activity (Fig. 4A; Supplemental Fig. S4A). When the marked skin was lineage-traced into anagen, we observed a gradual increase in marked adipocytes starting from Ana-III, when HF-TACs formed. At Ana-VI, $>30 \%$ of the mature adipocytes were marked (Fig. 4A; Supplemental Fig. S4B), suggesting that they are produced by Pdgfra-CreER ${ }^{+}$cells. Importantly, the numbers of newly generated adipocytes seen in Pdgfra-CreER; R26-mT/mG were similar to those observed with AdipoQ-CreER; R26- $\mathrm{mT} / \mathrm{mG}$ lineage tracing at each stage (Fig. 1B). These data suggest that PdgfraCreER marks the majority of adipocyte precursors that produce adipocytes in anagen.

Mx1-Cre is another inducible Cre that is normally inactive until administration of synthetic dsRNA (such as polyinosinic:polycytidylic acid [poly I:C]) to elevate interferon signaling. Upon poly I:C induction, Mx1-Cre is active in the bone marrow stroma (Park et al. 2012; Saez et al. 2014; Yue et al. 2016). Our analysis suggested that Mx1-Cre could also be used to target dermal cells but with interesting distinctions from Pdgfra-CreER: When Mx1-Cre; R26-1sl-YFP mice were induced in telogen, $M \times 1-C r e$ was detected in the dermal fibroblasts but not in mature adipocytes. Mx1-Cre was also detected in endothelial cells $\left(\mathrm{CD} 31^{+}\right)$and immune cells $\left(\mathrm{CD} 45^{+}\right)$. About $20 \%$ of the adipocyte precursors were also labeled in telogen. Interestingly, when Mx1-Cre; R26-1slYFP mice were lineage-traced into anagen, few if any mature adipocytes were $\mathrm{YFP}^{+}$, suggesting that the subset of adipocyte precursors marked specifically by Mx1-Cre is not the main contributor of adipogenesis in anagen (Fig. 4B; Supplemental Fig. S5). Thus, while PdgfraCreER marks adipocyte precursors that produce mature adipocytes in anagen, $M \times 1$-Cre does not. This important difference provides a possibility to assess gene function in adipogenic versus nonadipogenic fibroblasts. Moreover, Mx1-Cre also provides an opportunity to study gene function in endothelial cells and cells of hematopoietic origin in the skin. Our data also indicate a hitherto unrecognized heterogeneity among the adipocyte precursors in the dermis: a subset marked by Pdgfra-CreER that gives rise to mature adipocytes in anagen and a subset marked by Mx1-Cre that does not.

\section{SHH pathway activity is required in the adipocyte precursors for dermal adipogenesis}

We next used Pdgfra-CreER and Mx1-Cre to delete Smo in distinct subsets of dermal cells. When tamoxifen was applied to the Pdgfra-CreER; Smofl/fl mice during telogen, no significant changes in dermal adipocytes were observed in telogen or early anagen (Supplemental Fig. S6A,B). However, from Ana-III onward, Pdgfra-CreER; Smofl/fl mice displayed a thinner dermal adipose layer composed of significantly fewer mature adipocytes. Since the dermis failed to expand concomitantly, these anagen hair follicles in Pdgfra-CreER; Smofl/f1 mice were forced to bend in order to grow within a restricted space (Fig. 5A). In contrast, upon poly I:C induction, Mx1-Cre; Smofl/fl mice displayed normal adipose layer thickness in both telogen and anagen (Fig. 5B; data not shown). These results suggest that $S m o$ activity is likely to be directly required by adipocyte precursors to promote anagen-associated dermal adipogenesis. Furthermore, since Mx1-Cre is active in the cutaneous blood vessels and immune cells, the results also suggest that Smo activity is not required in the endothelial cells and cells of hematopoietic origin for dermal adipogenesis. 


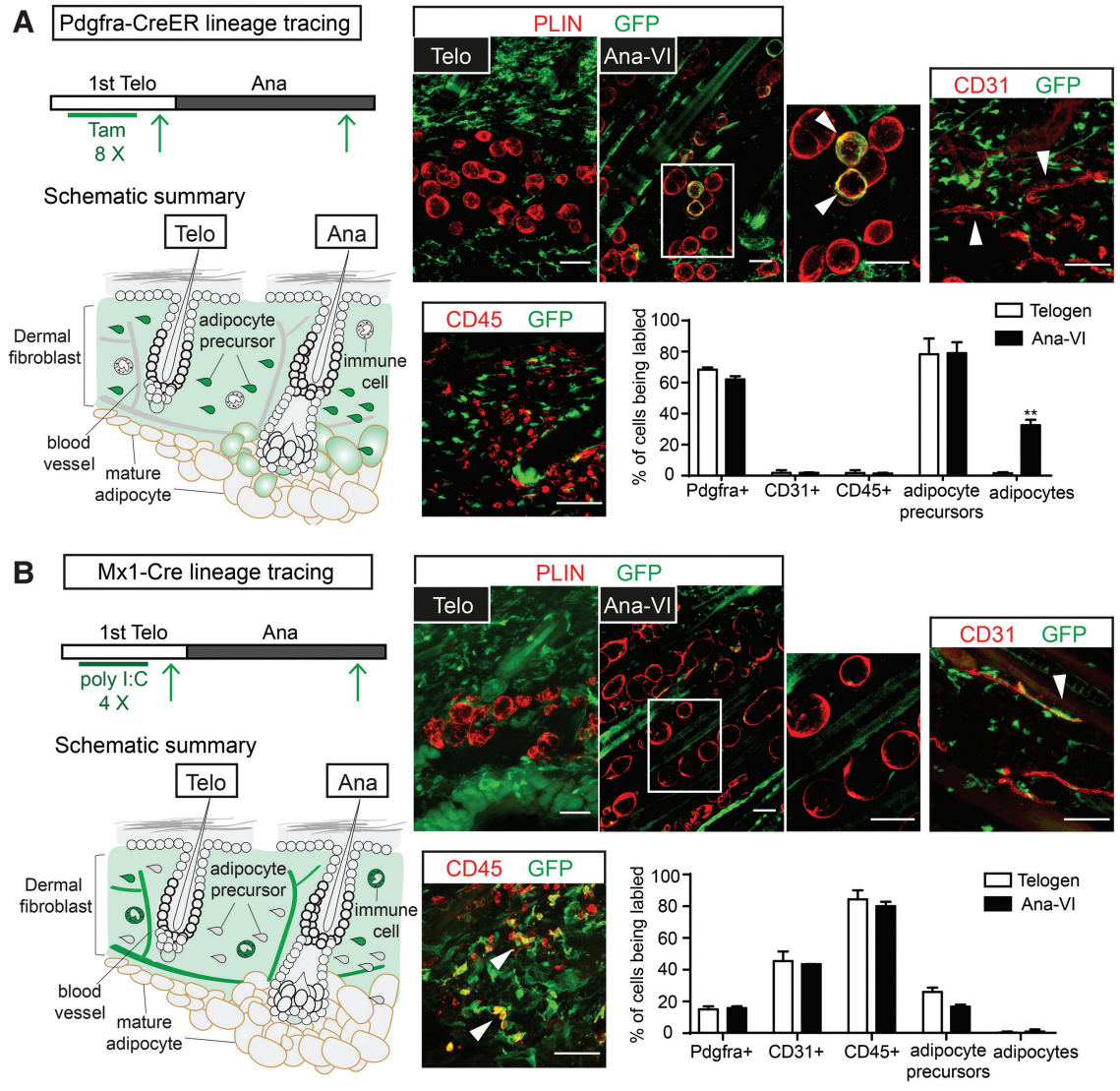

Figure 4. Pdgfra-CreER and Mx1-Cre mark distinct cell types in the dermis. $(A)$ Lineage tracing with Pdgfra-CreER to monitor Cre activity and progeny derived from Pdgfra-CreER ${ }^{+}$cells. Pdgfra-CreER was crossed to R26-1sl-YFP or R26-mT/mG mice. Tamoxifen was given at telogen. Skin biopsy samples were first taken after injection at telogen to determine cell types labeled by Pdgfra-CreER prior to anagen entry. Another skin sample was then taken at Ana-VI to monitor cell types that were labeled by Rosa reporters at the end of anagen. The schematics summarize the induction scheme and lineage-tracing results. Note that the number of labeled adipocytes increases significantly at anagen, suggesting that Pdgfra-CreER labels adipocyte precursors giving rise to mature adipocytes in anagen. $(B)$ Lineage tracing with Mx1-Cre; R261sl-YFP to monitor Cre activity and progeny derived from $\mathrm{Mx} 1-\mathrm{Cre}^{+}$cells. Polyinosinic: polycytidylic acid (poly I:C) was given to Mx1-Cre; R26-1sl-YFP mice at telogen. First, biopsy samples were taken at telogen after poly I:C injections, and another skin sample was taken at the end of anagen. The schematics summarize the induction scheme and lineage-tracing results. Images at the right show representative examples for labeled cells (stained as $\mathrm{GFP}^{+}$) together with markers of distinct dermal cell types. Immunofluorescence staining shows labeled cells $\left(\mathrm{GFP}^{+}\right)$in relation to markers of adipocytes (PLIN), endothelial cells (CD31), and immune cells (CD45). Bars quantify the percentage of cells being labeled for each cell type at different hair cycle stages. The percentages of labeled Pdgfra ${ }^{+}$(pan-fibroblasts: Pdgfra ${ }^{+}$, $\mathrm{CD} 31^{-}$, and $\mathrm{CD} 45^{-}$), CD31 ${ }^{+}$(endothelial cells), CD45 (pan-immune cells), and adipocyte precursors $\left(\mathrm{CD} 24^{+}, \mathrm{Scal}^{+}, \mathrm{CD}^{+} 1^{-}\right.$, and CD45 ${ }^{-}$) were quantified with FACS analysis. Mature adipocytes were quantified by counting the numbers of Perilipin- and GFP-double-positive cells among all Perilipin-positive cells in the dermis. $n=2$ mice for each genotype. Data are mean \pm SD. Bars, $50 \mu m$. $\left(^{* *}\right) P<0.01$.

To further test the autonomous requirement of $S m o$ in adipocyte precursors, we used mosaic analysis by treating Pdgfra-CreER; Smof1/f1; R26-1sl-YFP animals with a reduced dose of tamoxifen in telogen. In doing so, only a small subset of adipocyte precursors would be devoid of $S m o$ and marked by YFP. If Smo is required autonomously in the adipocyte precursors, we expect that YFP-positive adipocyte precursors cannot make mature adipocytes. As such, the adipocytes in the following anagen would all be YFP-negative. In contrast, if Smo is not required in the adipocyte precursors, we expect to see that both the wild-type and Smo knockout adipocyte precursors can contribute to mature adipocytes so that a fraction of mature adipocytes should be YFP-positive in anagen (Fig. 5C).

Reducing the tamoxifen dose led to fewer $\mathrm{YFP}^{+}$cells in the dermis, evident in the telogen skins from PdgfraCreER; Smofl/fl; R26-1sI-YFP and Pdgfra-CreER; R26-IslYFP controls (Fig. 5D,E; Supplemental Fig. S6C). When the Pdgfra-CreER; Smofl/f1; R26-1sl-YFP animals entered anagen, their adipose layers were composed exclusively of YFP-negative adipocytes. In contrast, in Pdgfra-CreER; R26-1sl-YFP controls, the anagen adipose layer was composed of both YFP-positive and YFP-negative adipocytes
(Fig. 5E,F). Importantly, in Pdgfra-CreER; Smofl/fl; R26lsl-YFP animals, the YFP-positive cells were indeed Smo knockout cells with reduced Gli1 levels, as indicated by RT-PCR (Fig. 5G). Other than the lack of Smo knockout $\left(\mathrm{YFP}^{+}\right)$adipocytes, Pdgfra-CreER; Smofl/fl; R26-Isl-YFP skin and hair follicles were indistinguishable from the controls (Fig. 5E; Supplemental Fig. S6D). Together, our data illustrate that SHH signaling is required in the adipocyte precursors autonomously. When the adipocyte precursors are devoid of Smo, they fail to respond to HFTAC-derived SHH in anagen and do not produce mature adipocytes even when hair follicle growth is normal.

\section{SHH pathway activity promotes adipocyte precursor proliferation in anagen}

We next aimed to identify how SHH might impact adipogenesis. EdU incorporation analysis on FACS-purified adipocyte precursors indicated that, in controls, adipocyte precursor proliferation increased at mid-anagen (Ana-III to Ana-IV) right after HF-TAC formation (Fig. 6A). Proliferation of adipocyte precursors was significantly impaired when Smo was knocked out with Pdgfra-CreER or when 
A

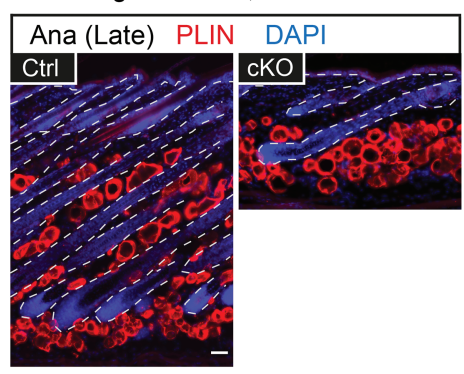

B

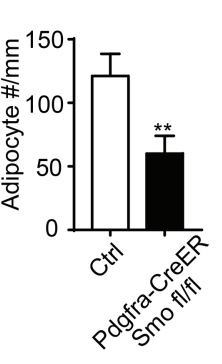

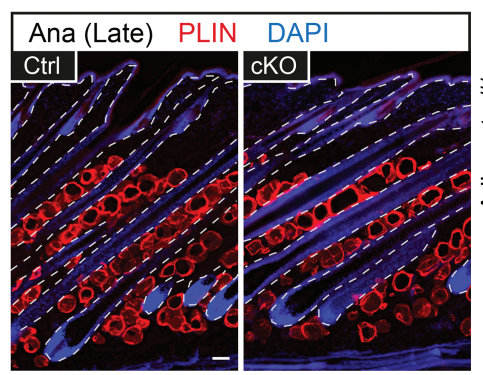

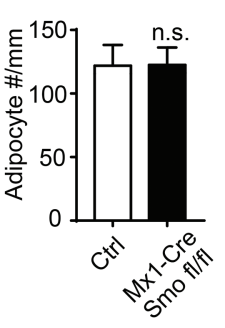

C Pdgfra-CreER; Smofl/fl; R26-YFPfl/+ E

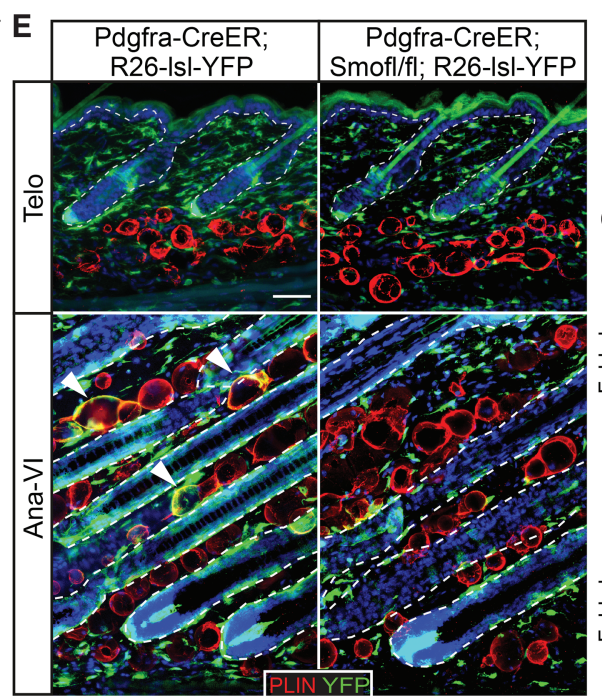
Smo required in Smo not required in adipocyte precursors adipocyte precursors

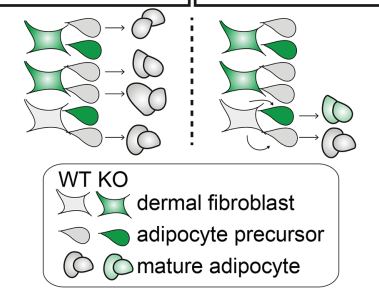

D \% of YFP+ adipocyte precursors in telogen

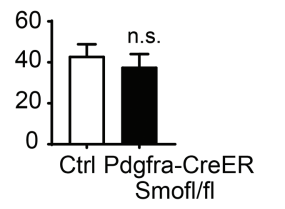

F \% of YFP+ adipocytes in Ana-VI

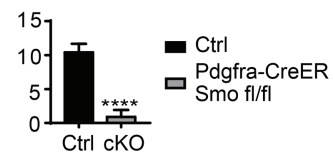

G

Smo levels in adipocyte precursors

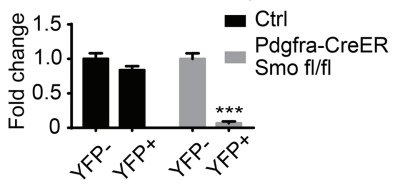

Gli1 levels in adipocyte precursors

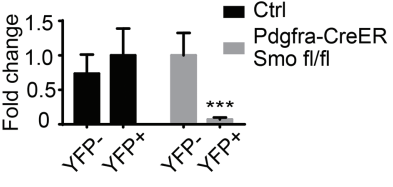

Figure 5. Deletion of $S m o$ in adipocyte precursors inhibits dermal adipogenesis. $(A, B)$ Full-anagen skin samples from controls, $P d g$ fraCreER; Smofl/f1 $(n=6)$, and Mx1-Cre; Smofl/f1 $(n=4)$ with immunolabeling of Perilipin and quantification of adipocyte numbers per millimeter width of skin. $(C-G)$ Mosaic analysis assessing the autonomous requirement of $S m o$ in adipocyte precursors. $(C, D)$ Reduction of tamoxifen treatment (four times in telogen) leads to partial activation of Pdgfra-CreER in a subset of adipocyte precursors, which is revealed as the $\mathrm{YFP}^{+}$subset. The percentages of $\mathrm{YFP}^{+}$adipocyte precursors right after tamoxifen pulse in control (Pdgfra-CreER; R26-lslYFP) and Pdgfra-CreER; R26-1sl-YFP; Smofl/f1 are similar, as shown in D. (E,F) The YFP ${ }^{+}$adipocytes in Pdgfra-CreER; R26-1sl-YFP control and Pdgfra-CreER; Smofl/f1; R26-lsl-YFP animals at the telogen and anagen stages are imaged and quantified. $n=3$. Note that while YFP ${ }^{+}$ adipocytes exist in Pdgfra-CreER; R26-1sl-YFP skin (arrowheads, black bar in F), few if any YFP ${ }^{+}$adipocytes are found in Pdgfra-CreER; Smofl/fl; R26-lsl-YFP skin (gray bar in F). (G) RT-PCR of Smo and Gli1 in YFP ${ }^{+}$and YFP $^{-}$adipocyte precursors purified from Pdgfra-CreER; R26-1sl-YFP control and Pdgfra-CreER; Smof1/f1; R26-1sl-YFP animals. Data are mean \pm SD. Bars, $50 \mu m .\left(^{* * * *}\right) P<0.0001 ;\left({ }^{* * *}\right) P<0.001 ;\left({ }^{* *}\right)$ $P<0.01$; (n.s.) not significant.

Shh was depleted from HF-TACs (Fig. 6A,B). Moreover, when low-dosage tamoxifen was given to Pdgfra-CreER; Smofl/fl; R26-lsl-YFP animals to conduct mosaic analysis, the Smo knockout adipocyte precursors displayed proliferation deficits compared with the controls (Fig. 6C). These data suggest that $\mathrm{SHH}$ signaling is required to promote adipocyte precursor proliferation.

We next investigated the molecular changes in Shh-or Smo-deficient skin. SHH is known to regulate proliferation through regulating cell cycle genes, including Dand E-type cyclins (Kenney and Rowitch 2000; DumanScheel et al. 2002). RT-PCR analysis indicated that known SHH targets Gli1 and Patched-1 (Ptch1) as well as cell cycle genes Cyclin D1 (Cond1), Cyclin D2 (Ccnd2), Cyclin E1 (Ccne1), Cyclin E2 (Ccne2), E2f1, and E2f2 were all significantly down-regulated in adipocyte precursors FACS-purified from K15-CrePGR; Shhfl/f1 or Pdgfra-CreER; Smofl/fl mice. In addition, Pparg, a master regulator of adipogenesis (Tontonoz and Spiegelman 2008), was also down-regulated in the adipocyte precursors devoid of SHH signaling (Fig. 6D,E). Of note, Gli1, Ptch1, Ccnd1, Ccnd2, and Pparg were all up-regulated in the adipocyte precursors FACS-purified from mid-anagen skin compared with those purified from telogen, consistent with the notion that $\mathrm{SHH}$ signaling is up-regulated when skin transitions from telogen to mid-anagen (Supplemental Fig. S7). Collectively, our data suggest that SHH secreted from HF-TACs promotes dermal adipogenesis by promoting dermal adipocyte precursor proliferation and regulating Pparg.

\section{Overexpression of SHH leads to further expansion of skin thickness}

Our data and previous work together support an intriguing model in which HF-TACs perform two critical 


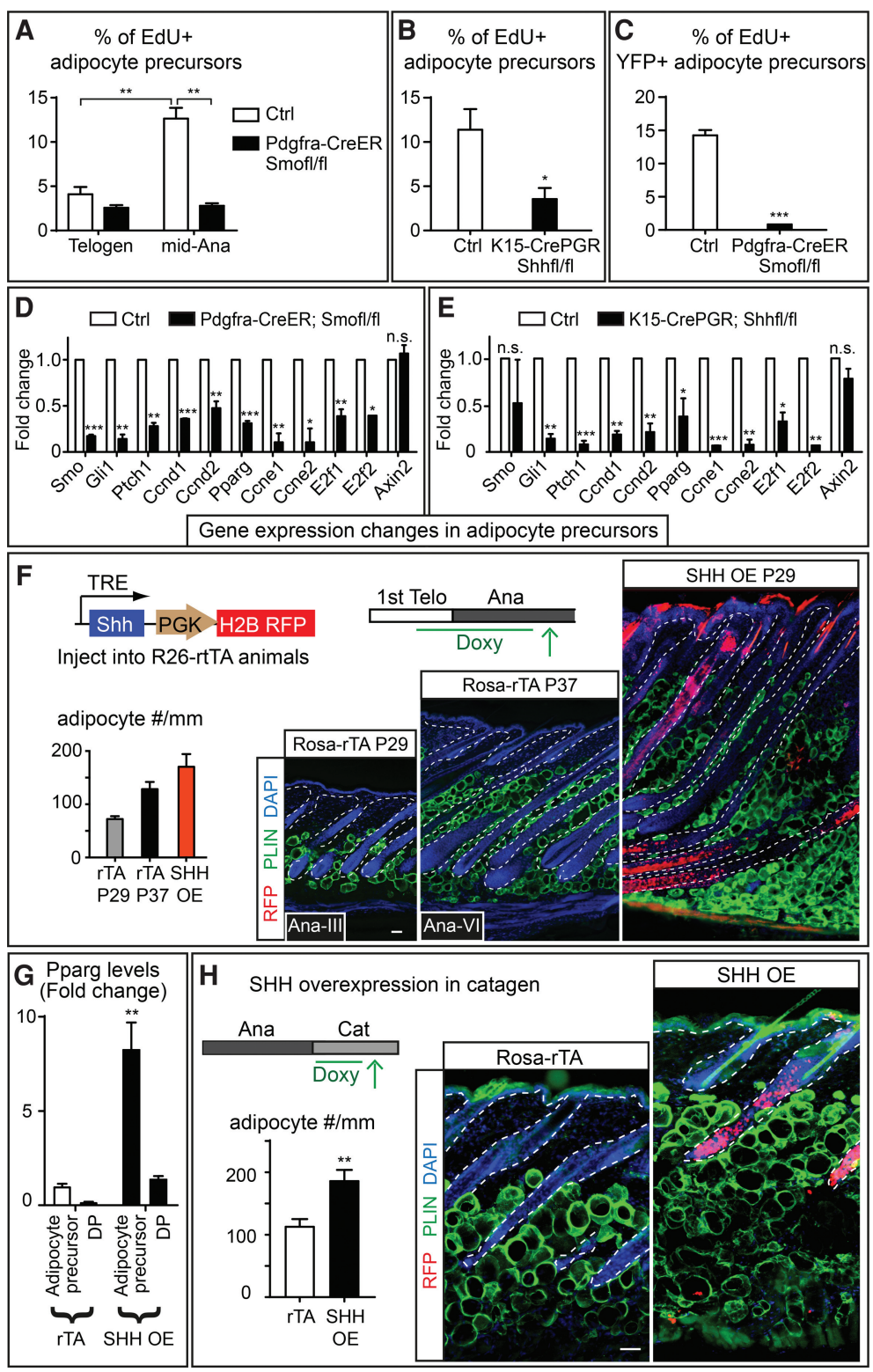

Figure 6. Reduction of SHH signaling inhibits adipocyte precursor proliferation, while overexpression of Shh promotes adipogenesis independently of hair cycle status. (A) Quantification of EdU ${ }^{+}$adipocyte precursors in control and Pdgfra-CreER; Smofl/f1 animals at different hair cycle stages. Adipocyte precursors were FACSpurified as Pdgfra ${ }^{+}, \mathrm{CD}_{2} 4^{+}, \mathrm{Sca}^{+}, \mathrm{CD} 31^{-}$and CD45 ${ }^{-}$. (B) Quantification of EdU ${ }^{+}$adipocyte precursors in control and K15-CrePGR; Shhfl/fl mice at mid-anagen. (C) Quantification of EdU $\mathrm{YFP}^{+}$adipocyte precursors in Pdgfra-CreER; R26-Isl-YFP control and Pdgfra-CreER; Smofl/f1; R26-1s1-YFP animals at mid-anagen after mosaic induction of Pdgfra-CreER (four tamoxifen injections). (D) RT-PCR analysis for gene expression changes in adipocyte precursors FACS-purified from control and Pdgfra-CreER; Smofl/fl animals at midanagen. (E) RT-PCR analysis for gene expression changes in adipocyte precursors purified from control and K15-CrePGR; Shhfl/fl animals at mid-anagen. $(F)$ Overexpression of SHH by transducing the skin with lentiviruses containing doxycycline (Doxy)-regulatable $\mathrm{SHH}$. Doxycycline was given from postnatal day 21 (P21) to P29. Both the littermate age control (RosarTA P29) and Ana-VI control (Rosa-rTA P37) are shown as comparisons with the $\mathrm{SHH}$ overexpression skin. The analysis of variance reveals a significant difference among these three groups. $F_{2,15}=47.18 . P<0.0001$, one-way ANOVA. $n=2$ mice for Rosa-rtTA controls; $n=3$ mice for $\mathrm{SHH}$ overexpression. (G) RT-PCR of Pparg in dermal papilla (DP) and adipocyte precursors FACS-purified from Rosa-rtTA control and SHH overexpression skins. $(H)$ Overexpression of $\mathrm{SHH}$ in catagen by transducing Rosa-rtTA mice with doxycycline-regulatable $\mathrm{SHH}$ as in $F$, but doxycycline was given after catagen entry for $5 \mathrm{~d}$. Data are mean \pm SD. Bars, 50 um. $\left(^{* * *}\right) P<0.001 ;\left({ }^{* *}\right) P<0.01 ;\left({ }^{*}\right) P<0.05$; (n.s.) not significant.

functions concurrently through SHH: (1) promoting hair follicle downgrowth and Bu-SC self-renewal (Hsu et al. 2014b) and (2) signaling to the surrounding dermis to promote adipogenesis. If this model is correct, overexpression of SHH should lead to further expansion of skin thickness by promoting hair follicle downgrowth and dermal adipogenesis concomitantly. To test this, we used in utero injection to introduce lentiviruses containing $p T R E-S h h$ into the skin epithelium of Rosa-rTA mice (Beronja et al. 2010). This approach allowed SHH to be expressed throughout the transduced epidermis and hair follicles upon doxycycline treatment. When Shh was induced to express at telogen, the hair follicles entered anagen immediately due to SHH's known role in promoting Bu-SCs activation (Hsu et al. 2014b). Interestingly, after $8 \mathrm{~d}$, the thickness of Shh-overexpressing skin was drastically thickened and nearly doubled compared with Ana-VI con- trol skin. Shh-overexpressing skin contained significantly more adipocytes, and the hair follicle length was also extended (Fig. 6F). RT-PCR of FACS-purified dermal populations suggested that Gli1 and Ptch1 were up-regulated in the adipocyte precursors and dermal papilla but not other cell types, suggesting that these two populations were more responsive to $\mathrm{SHH}$ than other dermal cells (Supplemental Fig. S8A-D). Interestingly, Pparg was significantly up-regulated in only the adipocyte precursors and not other dermal populations (Fig. 6G). Moreover, expression levels of Ccnd1 and E2f were also up-regulated in adipocyte precursors when Shh was overexpressed, suggesting that these adipocyte precursors became more proliferative (Supplemental Fig. S8E). These data further underscore the important role of $\mathrm{SHH}$ in modulating the regenerating tissue and restructuring the surrounding dermal environment in a coordinated fashion. 
To assess $\mathrm{SHH}^{\prime}$ s potency in initiating adipogenesis, we further evaluated the consequences when $\mathrm{SHH}$ is forced to express in catagen, a stage in which SHH-expressing HF-TACs are normally destroyed. To that end, we induced SHH expression after Rosa-rTA; pTRE-Shh mice entered catagen. Overexpression of SHH in catagen led to significantly more adipocyte production in catagen, as revealed by increased adipocyte numbers as well as increased adipocyte precursor proliferation (Fig. $6 \mathrm{H}$; Supplemental Fig. S8F). However, hair follicle destruction was not significantly perturbed. Together, these data suggest that expression of $\mathrm{SHH}$ at the stage when HF-TACs are destroyed is sufficient to reinitiate adipogenesis, underscoring $\mathrm{SHH}^{\prime}$ role in driving dermal adipogenesis.

\section{Hair follicle-derived SHH is critical for adipocyte formation in the embryonic dermis}

Last, we aimed to determine whether $\mathrm{SHH}$ is also essential for adipocyte formation in development or is required only for adipogenesis during the adult hair cycle. In the embryonic skin, adipocytes form de novo from dermal fibroblasts (Driskell et al. 2013). Hair follicles evaginate from the epidermis in three waves, and the majority of hair follicles forms during the second (embryonic day 16.5 [E16.5]) and the third (E18.5) waves (Fig. 7A). SHH expression appears at the leading edge of the developing placode and germ and is subsequently confined to the TACs of the peg stage hair follicles (Supplemental Fig. S9A; Woo et al. 2012; Ouspenskaia et al. 2016). We found that dermal adipocytes first appeared around E17.5 but only sparsely. Newly emerged adipocytes often clustered around the guard hairs (Supplemental Fig. S9B). Dermal adipocytes became more prominent at birth, which led to the formation of the adipocyte-rich hypodermis (Fig. 7A). Therefore, the timing of SHH expression coincides with adipocyte emergence in the developing skin, mirroring what we observed in the adult. In addition, overexpression of SHH in embryonic skin also led to more adipocyte production (Supplemental Fig. S9C) despite the fact that hair follicle development became abnormal (Oro et al. 1997; Ellis et al. 2003; Youssef et al. 2012).

We next knocked out Shh from the developing hair follicles using K14-Cre, which is expressed in the epidermal progenitors that give rise to all hair follicles. Consistent with previous findings (St-Jacques et al. 1998; Chiang et al. 1999), when Shh was depleted, the hair follicles were specified but failed to downgrow. Interestingly, we noticed a drastic reduction in the number of adipocytes in K14-Cre; Shhfl/f1 skin, suggesting that SHH is also essential for de novo formation of adipocytes in the developing dermis. The abrogated adipogenesis is not due to impaired hair follicle downgrowth or secondary signals elicited by $\mathrm{SHH}$ in the epidermal compartment, since K14-Cre; Smofl/f1 skin has severely retarded hair growth similar to Shh knockout (Gritli-Linde et al. 2007) but normal adipocyte formation at birth (Fig. 7B)

We next determined whether SHH pathway activity is required in dermal fibroblasts for adipocyte formation.
A Adipocyte development in the embryonic skin

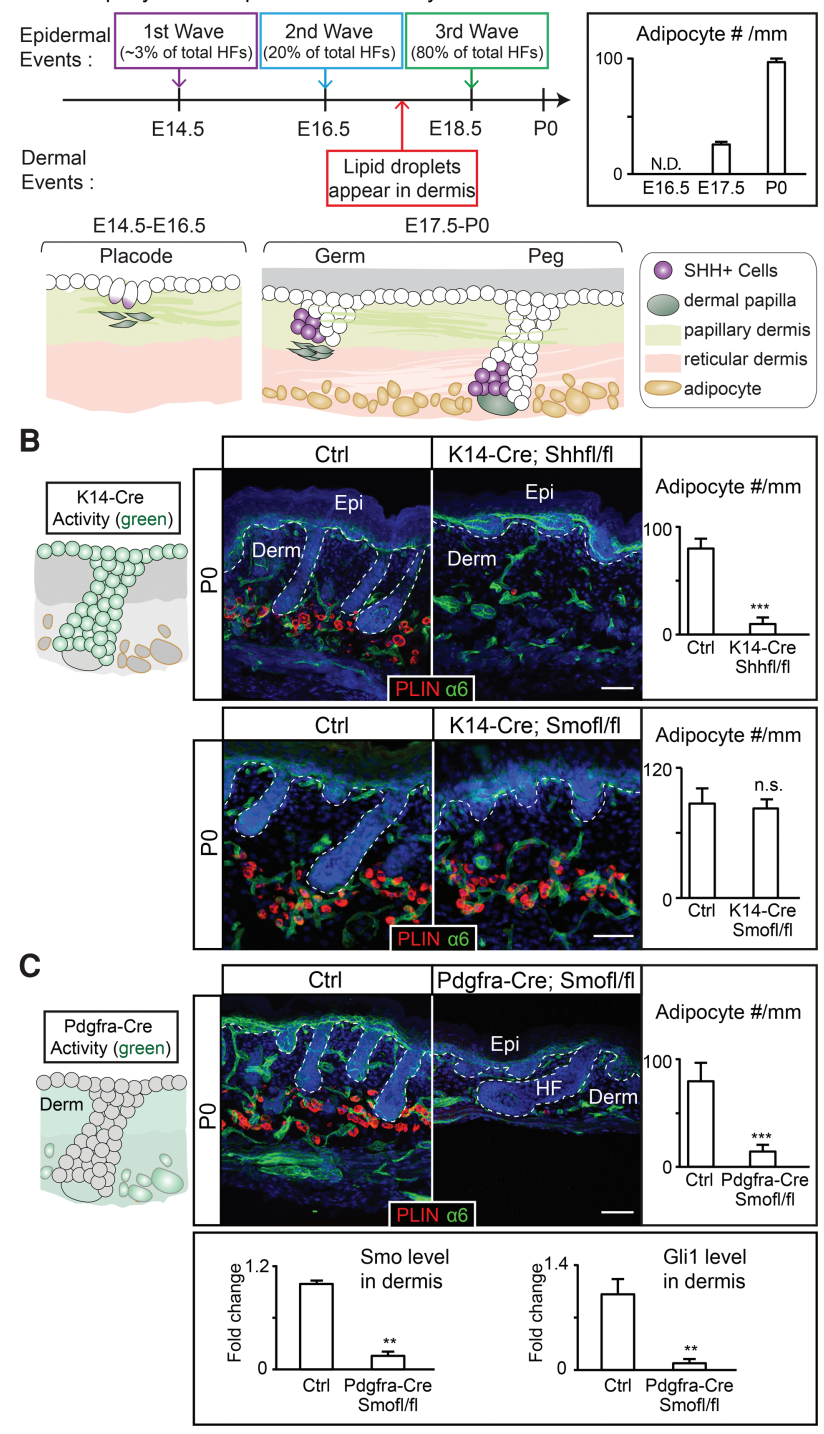

Figure 7. The SHH pathway is required for the de novo formation of dermal adipocytes during development. $(A)$ De novo formation of adipocytes in the embryonic dermis begins between the second and third wave of hair follicle development at E17.5. The schematic summarizes $\mathrm{SHH}^{+}$cells found in different stages (placode, germ, and peg stages) of hair follicles during development. The bar graph summarizes the number of Perilipin ${ }^{+}$cells per millimeter width of skin at different embryonic/postnatal days. (N.D.) Not determined. $n=3$ mice for each stage. $(B)$ Deletion of Shh from the embryonic epidermis and hair follicles by K14-Cre prevents dermal adipocyte formation, while deletion of Smo using K14-Cre results in changes in hair follicle length but no changes in adipocyte formation. Cells derived from K14-Cre ${ }^{+}$ embryonic progenitors are denoted in green in the schematic. Bars represent the number of Perlipin ${ }^{+}$cells per millimeter width of skin. $n=4$. (C) Schematic showing cells derived from Pdgfra$\mathrm{Cre}^{+}$cells at P0. Deleting Smo using Pdgfra-Cre leads to severe deficits in dermal adipocyte formation. The bar graphs represent the number of Perilipin ${ }^{+}$cells per millimeter width of skin and RT-PCR of Smo and Gli1 in FACS-purified Pdgfra ${ }^{+}$cells. $n=4$.

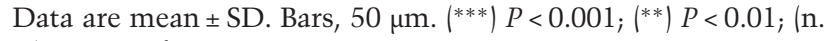
s.) not significant. 
For this, we used Pdgfra-Cre (Roesch et al. 2008) to deplete Smo. Our analysis suggested that Pdgfra-Cre activity is active in the embryonic dermis as early as E12.5 (data not shown). In Pdgfra-Cre; R26-mT/mG mice, dermal fibroblast-derived cells, including papillary dermis, reticular dermis, and dermal adipocytes, are all positive for GFP by postnatal day 0 (P0). Pdgfra-Cre is not active in blood vessels, immune cells, the epidermis, or hair follicles (Supplemental Fig. S9D). Pdgfra-Cre; Smofl/f1 skin displayed profound defects in adipogenesis and efficient depletion of Smo and Gli1 in the dermis (Fig. 7C). Nevertheless, hair follicle growth was not altered as severely in the Pdgfra-Cre; Smofl/fl skin as in K14-Cre; Shhfl/fl or K14-Cre; Smo fl/f1 skin. Proliferation was decreased in the embryonic lower dermal layer (which gives rise to the adipocytes) (Driskell et al. 2013) when either Shh was depleted from the embryonic hair follicles or Smo was knocked out from the dermis (Supplemental Fig. S9E). Apoptosis in the dermis was not altered (Supplemental Fig. S9F). Collectively, our data demonstrate that, similar to the adult stage, dermal adipocyte formation also requires active $\mathrm{SHH}$ signaling.

\section{Discussion}

The development and maintenance of a fully functional organ rely on coordinated behaviors of different lineages. We are only beginning to discover the principles behind these synchronized actions among tissues. Coordination between two lineages can occur at the stem cell level, where two different types of adult stem cells located in a shared niche communicate with each other. For example, in the bulge, hair follicle stem cells influence melanocyte stem cells (Nishimura et al. 2010; Rabbani et al. 2011; Chang et al. 2013). Examples in which somatic stem cells induce changes in their neighboring cells have also been reported. For instance, in zebrafish, hematopoietic stem cells (HSCs) trigger the endothelial cells to wrap around them (Tamplin et al. 2015). In mice, HSCs delay vascular regeneration but promote blood vessel integrity in the bone marrow after irradiation (Zhou et al. 2015). These intriguing communications are likely tailored to the special needs of different stem cells.

One common challenge among all regenerating tissues is that their rapid growth often requires concurrent changes in the surrounding cell types for structural or nutritional support. However, stem cells are often not the major contributor to tissue production. In many tissues, including hair follicles, hematopoietic lineages, intestines, corneal epithelium, teeth, nerve systems, and certain cancers, stem cells proliferate sparingly to generate TACs as a transitional population (Diaz-Flores et al. 2006; Zhou et al. 2006; Li et al. 2012; Hoggatt et al. 2013; Hsu et al. 2014b; Ritsma et al. 2014). In sharp contrast to their relatively quiescent stem cell counterparts, TACs divide rapidly and generate large numbers of downstream progeny that form the bulk of the new tissue. In this sense, TACs are at an ideal juncture to orchestrate changes to the surrounding tissues that are tailored tightly to the needs of an actively regenerating tissue.
Here, we found that HF-TACs are an essential cell type that couples hair follicle growth with dermal expansion. This coordination allows the skin to make the changes necessary to accommodate the rapidly downgrowing hair follicles. Our findings here uncover the molecular nature, signaling source, and signal-receiving cells that are critical for this coupling event, resolving the long-standing question of how hair follicle growth and dermal adipose layer expansion can be tightly linked (Supplemental Fig. S10). The ability of TACs to mediate concurrent changes of surrounding tissues is likely a shared principle among many different systems due to TACs' common functions in producing large amounts of downstream progeny.

Both the anti-adipogenic and proadipogenic functions of the SHH pathway have been proposed: Cell culture studies show that $\mathrm{SHH}$ activation inhibits adipocyte differentiation while blocking the pathway-stimulated adipogenesis (Spinella-Jaegle et al. 2001; Suh et al. 2006; James et al. 2010; Fleury et al. 2016). HH pathways inhibit fat formation in Drosophila, and a conserved function has been suggested for certain white adipose tissues in mammals (Suh et al. 2006; Pospisilik et al. 2010). In contrast, elevated SHH levels in medulloblastoma lead to pronounced adipocyte generation, and the resulting tumors are filled with adipocytes (Bhatia et al. 2011). These studies suggest that $\mathrm{SHH}$ signaling can have a context-dependent function in adipogenesis depending on cell types, tissues, and culturing conditions. The dermal adipogenesis described here is a highly regulated process that occurs with a precise and predictable timing, which might have interesting differences from pathologically related adipogenesis or adipogenesis in culture. This context-dependent nature further underscores the importance of using cell type-specific manipulation such as that used here to study adipogenesis in a tissue-specific manner.

Mesenchymal cells, also referred to as the stroma, constitute the supportive structure for essentially all organs (Mendez-Ferrer et al. 2010; Rinkevich et al. 2015; Yue et al. 2016). Adipocytes are common components in the stroma and have emerged as important players within the stem cell niches in multiple organs. For example, in skin, dermal adipocytes and adipocyte precursors influence hair cycle progression (Plikus et al. 2008; Festa et al. 2011; Keyes et al. 2013). In addition, dermal adipocytes secrete antimicrobial peptides to protect the skin from bacterial infection (Zhang et al. 2015). In bone marrow, adipocyte numbers correlate inversely with hematopoiesis (Naveiras et al. 2009; Omatsu et al. 2014). In skeletal muscle, a group of $\mathrm{Pdgfa}^{+}$fibro/adipogenic progenitors (FAPs) is activated upon injury and can enhance satellite cell proliferation (Joe et al. 2010; Uezumi et al. 2010). It is worth noting that adipocytes are much larger and can change shape and size more readily than other cell types. These unique features make them ideal "fillers" in adult mammalian organs, which are often relatively constrained in their overall size. Growth or degeneration of one lineage is often compensated for by altering the numbers of adipocytes residing in the stroma of the same organ. While systemic factors such as leptin 
have been shown to influence adipocytes in the bone marrow (Yue et al. 2016), the local signals that control these dynamic changes in adipocytes within different mesenchymes remain largely unknown. It will be interesting to determine whether hematopoiesis, muscle repair, or other tissue regeneration processes also impact adipogenesis in the underlying stroma and to what extent the function of $\mathrm{SHH}$ in adipogenesis that we uncovered here is conserved.

In the past, TACs have been viewed as a passive transit population whose sole role is to produce tissues. Recent studies have begun to reveal the diverse functions of TACs, including feedback regulation to the stem cells (Hsu et al. 2014b). Our study further reveals an essential function of TACs in niche remodeling that is conducive to tissue regeneration, suggesting that TACs of one lineage can coordinate tissue production of a neighboring lineage. Given that TACs are found in many mammalian tissues and play an integral role in tissue regeneration, elucidating TACs' biology and function will continue to be critical for understanding and harnessing the full power of this multifaceted population.

\section{Materials and methods}

\section{Mice and constructs}

K15-CrePGR (Ito et al. 2005), K14-Cre (Dassule et al. 2000), Smofl/f1 (Long et al. 2001), Shhfl/f1 (Lewis et al. 2001), R26-1s1YFP (Srinivas et al. 2001), R26-rTA (Hochedlinger et al. 2005), R26-mTmG (Muzumdar et al. 2007), Pdgfra-Cre (Roesch et al. 2008), Pdgfra-CreER (Kang et al. 2010), and AdipoQ-CreER (Jeffery et al. 2014) were described previously and were obtained from The Jackson Laboratory. Mx1-Cre was obtained from Dr. David Scadden's laboratory (Harvard University). The Shh-overexpressing lentiviral construct (LV-TRE-Shh-PGK-H2BGFP) was a gift of Dr. Elaine Fuchs and was described previously (Hsu et al. 2014b). K15-CrePGR was activated by topical application of RU486 (4\% in ethanol). Pdgfra-CreER and AdipoQ-CreER were induced by intraperitoneal (i.p.) injection with $20 \mathrm{mg} / \mathrm{mL}$ tamoxifen for the amount of time indicated for each experiment. $\mathrm{EdU}(25 \mathrm{mg} / \mathrm{g})$ was injected for either $2 \mathrm{~h}$ in pregnant mice (i.p) or newborn pups (subcutaneous) or $4 \mathrm{~h}$ in adult mice (i.p.) before lethal administration of $\mathrm{CO}_{2}$. Rosa-rTA was activated by feeding mice with $200 \mathrm{mg} / \mathrm{kg}$ doxycycline chow or $300 \mu \mathrm{L}$ of $10 \mathrm{mg} / \mathrm{mL}$ gavage. Ultrasound-guided lentiviral injection procedures were performed as described previously (Beronja et al. 2010). All animals were maintained in an Association for Assessment and Accreditation of Laboratory Animal Care-approved animal facility at Harvard University, and procedures were performed with Institutional Animal Care and Use Committee-approved protocols.

\section{Hair cycle}

Hair cycle was determined as described previously (Muller-Rover et al. 2001). Since hair cycles vary among strains and sexes, stages instead of exact mouse ages were carefully evaluated for each experiment. The depilation experiment presented in Supplemental Figure 2D was performed during the second telogen, while the rest of the adult animals were analyzed at their synchronized first adult hair cycle. Mice of matched sex were analyzed. Cre, CreER alone, rtTA alone, or flox allele alone littermates were used as controls and subjected to the same treatment as the knockout an- imals. Although only sex-matched littermates were used for comparison, all phenotypes were observed in both males and females. For animals used for FACS, skin biopsies from the same animals were also taken for histology analysis to determine the hair cycle substages.

\section{FACS}

Mouse back skin from E17.5 embryos, P0 pups, or adults was dissected and treated with collagenase in HBSS for 20-30 min at $37^{\circ}$ $\mathrm{C}$ on an orbital shaker. The dermal fraction was collected by surgical knife scraping followed by centrifugation at $300 \mathrm{~g}$ for $10 \mathrm{~min}$. Dermal single-cell suspensions were obtained after $0.25 \%$ Trypsin treatment for $10-20 \mathrm{~min}$ at $37^{\circ} \mathrm{C}$ followed by filtering and centrifugation. Samples were stained for $30 \mathrm{~min}$ on ice. The following antibodies were used for cell sorting: Pdgfra-biotin (1:250; eBiosciences), CD45-PE-Cy7 (1:250; eBiosciences), CD31PE-Cy7 (1:200; eBiosciences), Sca1-PerCP-Cy5.5 (1:1000; eBiosciences), Dlk-FITC (1:250; MBL international), CD24-FITC (1:250; eBiosciences), CD24-PE (1:250; eBiosciences), and Sterpavidin-APC (1:500; eBiosciences). DAPI was used to exclude dead cells. Cells were FACS-sorted by BD-Aria sorters, and the data were analyzed by FlowJo.

\section{Histology and immunohistochemistry}

Prefixed (4\% in PFA) OCT-embedded embryos or skin samples were sectioned at the desired thickness (10-40 $\mu \mathrm{m})$. Immunohistochemistry was performed on sectioned slides by primary antibody incubation overnight at $4^{\circ} \mathrm{C}$ followed by secondary antibody staining for $1-4 \mathrm{~h}$ at room temperature. The following antibodies and dilutions were used: active-Caspase-3 (rabbit; 1:600; Cell Signaling Technology), GFP (rabbit; 1:400; Abcam), Pdgfra (goat; 1:200; R\&D), Dlk (goat; 1:200; R\&D), Perilipin (goat; 1:800; Abcam), CD31 (rat; 1:100; eBioscience), and CD45 (rat; 1:100; eBioscience). The secondary antibodies used were donkey anti-goat, rabbit, or rat conjugated with Alexa 488, 549, or 647 (1:800; Jackson Immunoresearch), respectively. EdU Click-It reaction was performed for $1 \mathrm{~h}$ at room temperature according to the manufacturer's instructions (Life Technologies). Samples were mounted in Prolong Gold with DAPI (Life Technologies).

\section{Quantitative real-time PCR}

FACS-isolated populations or adipocytes (centrifuge at $300 \mathrm{~g}$ for 5 min, the adipocytes were floating at the top) were lysed in Trizol LS (Thermo Fisher Scientific). RNAs were extracted using either Direct-Zol minipreparation or micropreparation kit (Zymo Research) according to the manufacturer's protocols. Purified total RNAs were quantified by a Nanodrop (Thermo Fisher Scientific) and stored at $-80^{\circ} \mathrm{C}$ for further analysis. The cDNA library was synthesized and prepared by SuperScript III reverse transcriptase with Oligo-dT according to the manufacturer's manual (Thermo Fisher Scientific). Quantitative PCR was performed by Power SYBR Green PCR master mix with the primers annealed to the genes of interest, and the $C_{\mathrm{t}}$ value was normalized using $\beta$-actin or Ppib2 primers.

\section{Confocal and epifluorescence microscope image processing}

Images were obtained with a Keyence epifluorescence microscope (Keyence America, BX-700) with a $10 \times$ or $20 \times$ objective with represented Z-plane-stacked images or with Zeiss LSM 510,700 , or 880 laser-scanning microscopes with a $20 \times$ air objective or a $40 \times$ oil-based objective (Carl Zeiss MicroImaging). 
Images were further processed and assembled into panels using Adobe Photoshop CS5 and Adobe Illustrator CS5.

\section{Statistical analyses}

Statistical significance between two groups in the figures was noted by asterisks $\left(P<0.05\left[{ }^{*}\right], P<0.01\left[{ }^{* *}\right], P<0.001\left[{ }^{* * *}\right]\right.$, and $P$ $<0.0001[* * * *])$. The data are presented as mean $\pm S D$, and statistical analysis was performed using unpaired two-tailed Student's $t$ test. Analyses of multiple groups were performed using one-way ANOVA.

\section{Acknowledgments}

We are grateful to David Scadden, Elaine Fuchs, and many colleagues who donated mice to The Jackson Laboratory (JAX) for providing mice and reagents; Joyce Lavecchio and Silvia Ionescu (Harvard Department of Stem Cell and Regenerative Biology Flow Cytometry Core) for sorting; Doug Richardson (Harvard Center for Biological Imaging) for advice on imaging; Harvard's Office of Animal Resources (OAR) for veterinary care; Yick W. Fong, Jayaraj Rajagopal, Lev Silberstein, and Liam Gaynor for comments on the manuscript; and members of Y.-C.H.'s laboratory, in particular Luke Demas and Sekyu Choi, for discussions and assistance with experiments. M.G.-C. was supported by the la Caixa Foundation fellowship and is a recipient of the Simmons Family Award. C.N.P. was supported by an EMBO fellowship (ALTF 552-2012). This work was supported by grants from the National Institutes of Health/National Institute of Arthritis and Musculoskeletal and Skin Diseases (R00-AR063127 and R01-AR070825 to Y.-C.H., and R01-AR063724 to E.E.). This work was also supported by the Smith Family Awards Program, Basil O'Connor Starter Scholar award, Harvard Stem Cell Institute seed grant, and Milton Fund (all to Y.-C.H.).

\section{References}

Beronja S, Livshits G, Williams S, Fuchs E. 2010. Rapid functional dissection of genetic networks via tissue-specific transduction and RNAi in mouse embryos. Nat Med 16: 821-827.

Bhatia B, Hsieh M, Kenney AM, Nahle Z. 2011. Mitogenic Sonic hedgehog signaling drives E2F1-dependent lipogenesis in progenitor cells and medulloblastoma. Oncogene 30: 410-422.

Blanpain C, Fuchs E. 2009. Epidermal homeostasis: a balancing act of stem cells in the skin. Nat Rev Mol Cell Biol 10: 207-217.

Brownell I, Guevara E, Bai CB, Loomis CA, Joyner AL. 2011. Nerve-derived sonic hedgehog defines a niche for hair follicle stem cells capable of becoming epidermal stem cells. Cell Stem Cell 8: 552-565.

Chang CY, Pasolli HA, Giannopoulou EG, Guasch G, Gronostajski RM, Elemento O, Fuchs E. 2013. NFIB is a governor of epithelial-melanocyte stem cell behaviour in a shared niche. Nature 495: 98-102.

Chen CC, Plikus MV, Tang PC, Widelitz RB, Chuong CM. 2016. The modulatable stem cell niche: tissue interactions during hair and feather follicle regeneration. I Mol Biol 428: 1423-1440.

Chiang C, Swan RZ, Grachtchouk M, Bolinger M, Litingtung Y, Robertson EK, Cooper MK, Gaffield W, Westphal H, Beachy PA, et al. 1999. Essential role for Sonic hedgehog during hair follicle morphogenesis. Dev Biol 205: 1-9.
Dassule HR, Lewis P, Bei M, Maas R, McMahon AP. 2000. Sonic hedgehog regulates growth and morphogenesis of the tooth. Development 127: 4775-4785.

Deschene ER, Myung P, Rompolas P, Zito G, Sun TY, Taketo MM, Saotome I, Greco V. 2014. $\beta$-Catenin activation regulates tissue growth non-cell autonomously in the hair stem cell niche. Science 343: 1353-1356.

Diaz-Flores L Jr, Madrid JF, Gutierrez R, Varela H, Valladares F, Alvarez-Arguelles H, Diaz-Flores L. 2006. Adult stem and transit-amplifying cell location. Histol Histopathol 21: 995-1027.

Donati G, Proserpio V, Lichtenberger BM, Natsuga K, Sinclair R, Fujiwara H, Watt FM. 2014. Epidermal Wnt/ $\beta$-catenin signaling regulates adipocyte differentiation via secretion of adipogenic factors. Proc Natl Acad Sci 111: E1501-E1509.

Driskell RR, Lichtenberger BM, Hoste E, Kretzschmar K, Simons BD, Charalambous M, Ferron SR, Herault Y, Pavlovic G, Ferguson-Smith AC, et al. 2013. Distinct fibroblast lineages determine dermal architecture in skin development and repair. Nature 504: 277-281.

Duman-Scheel M, Weng L, Xin S, Du W. 2002. Hedgehog regulates cell growth and proliferation by inducing Cyclin D and Cyclin E. Nature 417: 299-304.

Ellis T, Smyth I, Riley E, Bowles J, Adolphe C, Rothnagel JA, Wicking C, Wainwright BJ. 2003. Overexpression of Sonic Hedgehog suppresses embryonic hair follicle morphogenesis. Dev Biol 263: 203-215.

Festa E, Fretz J, Berry R, Schmidt B, Rodeheffer M, Horowitz M, Horsley V. 2011. Adipocyte lineage cells contribute to the skin stem cell niche to drive hair cycling. Cell 146: 761-771.

Fleury A, Hoch L, Martinez MC, Faure H, Taddei M, Petricci E, Manetti F, Girard N, Mann A, Jacques C, et al. 2016. Hedgehog associated to microparticles inhibits adipocyte differentiation via a non-canonical pathway. Sci Rep 6: 23479.

Greco V, Chen T, Rendl M, Schober M, Pasolli HA, Stokes N, Dela Cruz-Racelis J, Fuchs E. 2009. A two-step mechanism for stem cell activation during hair regeneration. Cell Stem Cell 4: 155-169.

Gritli-Linde A, Hallberg K, Harfe BD, Reyahi A, Kannius-Janson M, Nilsson J, Cobourne MT, Sharpe PT, McMahon AP, Linde A. 2007. Abnormal hair development and apparent follicular transformation to mammary gland in the absence of hedgehog signaling. Dev Cell 12: 99-112.

Hochedlinger K, Yamada Y, Beard C, Jaenisch R. 2005. Ectopic expression of Oct-4 blocks progenitor-cell differentiation and causes dysplasia in epithelial tissues. Cell 121: 465-477.

Hoggatt J, Mohammad KS, Singh P, Hoggatt AF, Chitteti BR, Speth JM, Hu P, Poteat BA, Stilger KN, Ferraro F, et al. 2013. Differential stem- and progenitor-cell trafficking by prostaglandin E2. Nature 495: 365-369.

Hsu YC, Pasolli HA, Fuchs E. 2011. Dynamics between stem cells, niche, and progeny in the hair follicle. Cell 144: 92-105.

Hsu YC, Li L, Fuchs E. 2014a. Emerging interactions between skin stem cells and their niches. Nat Med 20: 847-856.

Hsu YC, Li L, Fuchs E. 2014b. Transit-amplifying cells orchestrate stem cell activity and tissue regeneration. Cell 157: 935-949.

Ito M, Liu Y, Yang Z, Nguyen J, Liang F, Morris RJ, Cotsarelis G. 2005. Stem cells in the hair follicle bulge contribute to wound repair but not to homeostasis of the epidermis. Nat Med 11: 1351-1354.

James AW, Leucht P, Levi B, Carre AL, Xu Y, Helms JA, Longaker MT. 2010. Sonic Hedgehog influences the balance of osteogenesis and adipogenesis in mouse adipose-derived stromal cells. Tissue Eng Part A 16: 2605-2616. 
Jeffery E, Berry R, Church CD, Yu S, Shook BA, Horsley V, Rosen ED, Rodeheffer MS. 2014. Characterization of Cre recombinase models for the study of adipose tissue. Adipocyte 3: 206-211.

Joe AW, Yi L, Natarajan A, Le Grand F, So L, Wang J, Rudnicki MA, Rossi FM. 2010. Muscle injury activates resident fibro/ adipogenic progenitors that facilitate myogenesis. Nat Cell Biol 12: 153-163.

Kang SH, Fukaya M, Yang JK, Rothstein JD, Bergles DE. 2010. $\mathrm{NG}_{2}{ }^{+} \mathrm{CNS}$ glial progenitors remain committed to the oligodendrocyte lineage in postnatal life and following neurodegeneration. Neuron 68: 668-681.

Kenney AM, Rowitch DH. 2000. Sonic hedgehog promotes G(1) cyclin expression and sustained cell cycle progression in mammalian neuronal precursors. Mol Cell Biol 20: 9055-9067.

Keyes BE, Segal JP, Heller E, Lien WH, Chang CY, Guo X, Oristian DS, Zheng D, Fuchs E. 2013. Nfatcl orchestrates aging in hair follicle stem cells. Proc Natl Acad Sci 110: E4950-E4959.

Kretzschmar K, Weber C, Driskell RR, Calonje E, Watt FM. 2016. Compartmentalized epidermal activation of $\beta$-catenin differentially affects lineage reprogramming and underlies tumor heterogeneity. Cell Rep 14: 269-281.

Lewis PM, Dunn MP, McMahon JA, Logan M, Martin JF, St-Jacques B, McMahon AP. 2001. Cholesterol modification of sonic hedgehog is required for long-range signaling activity and effective modulation of signaling by Ptc1. Cell 105: 599-612.

Li CY, Cha W, Luder HU, Charles RP, McMahon M, Mitsiadis TA, Klein OD. 2012. E-cadherin regulates the behavior and fate of epithelial stem cells and their progeny in the mouse incisor. Dev Biol 366: 357-366.

Lichtenberger BM, Mastrogiannaki M, Watt FM. 2016. Epidermal $\beta$-catenin activation remodels the dermis via paracrine signalling to distinct fibroblast lineages. Nat Commun 7: 10537.

Long F, Zhang XM, Karp S, Yang Y, McMahon AP. 2001. Genetic manipulation of hedgehog signaling in the endochondral skeleton reveals a direct role in the regulation of chondrocyte proliferation. Development 128: 5099-5108.

Mendez-Ferrer S, Michurina TV, Ferraro F, Mazloom AR, Macarthur BD, Lira SA, Scadden DT, Ma'ayan A, Enikolopov GN, Frenette PS. 2010. Mesenchymal and haematopoietic stem cells form a unique bone marrow niche. Nature 466: 829-834.

Muller-Rover S, Handjiski B, van der Veen C, Eichmuller S, Foitzik K, McKay IA, Stenn KS, Paus R. 2001. A comprehensive guide for the accurate classification of murine hair follicles in distinct hair cycle stages. J Invest Dermatol 117: 3-15.

Muzumdar MD, Tasic B, Miyamichi K, Li L, Luo L. 2007. A global double-fluorescent Cre reporter mouse. Genesis 45: 593-605.

Naveiras O, Nardi V, Wenzel PL, Hauschka PV, Fahey F, Daley GQ. 2009. Bone-marrow adipocytes as negative regulators of the haematopoietic microenvironment. Nature 460: 259-263.

Nishimura EK, Suzuki M, Igras V, Du J, Lonning S, Miyachi Y, Roes J, Beermann F, Fisher DE. 2010. Key roles for transforming growth factor $\beta$ in melanocyte stem cell maintenance. Cell Stem Cell 6: 130-140.

Omatsu Y, Seike M, Sugiyama T, Kume T, Nagasawa T. 2014. Foxc1 is a critical regulator of haematopoietic stem/progenitor cell niche formation. Nature 508: 536-540.

Oro AE, Higgins KM, Hu Z, Bonifas JM, Epstein EH Jr, Scott MP. 1997. Basal cell carcinomas in mice overexpressing sonic hedgehog. Science 276: 817-821.

Ouspenskaia T, Matos I, Mertz AF, Fiore VF, Fuchs E. 2016. WNT-SHH antagonism specifies and expands stem cells prior to niche formation. Cell 164: 156-169.
Park D, Spencer JA, Koh BI, Kobayashi T, Fujisaki J, Clemens TL, Lin CP, Kronenberg HM, Scadden DT. 2012. Endogenous bone marrow MSCs are dynamic, fate-restricted participants in bone maintenance and regeneration. Cell Stem Cell 10: 259-272.

Plikus MV, Mayer JA, de la Cruz D, Baker RE, Maini PK, Maxson R, Chuong CM. 2008. Cyclic dermal BMP signalling regulates stem cell activation during hair regeneration. Nature 451: 340-344.

Pospisilik JA, Schramek D, Schnidar H, Cronin SJ, Nehme NT, Zhang X, Knauf C, Cani PD, Aumayr K, Todoric I, et al. 2010. Drosophila genome-wide obesity screen reveals hedgehog as a determinant of brown versus white adipose cell fate. Cell 140: 148-160.

Rabbani P, Takeo M, Chou WC, Myung P, Bosenberg M, Chin L, Taketo MM, Ito M. 2011. Coordinated activation of wnt in epithelial and melanocyte stem cells initiates pigmented hair regeneration. Cell 145: 941-955.

Rinkevich Y, Walmsley GG, Hu MS, Maan ZN, Newman AM, Drukker M, Januszyk M, Krampitz GW, Gurtner GC, Lorenz $\mathrm{HP}$, et al. 2015. Skin fibrosis. Identification and isolation of a dermal lineage with intrinsic fibrogenic potential. Science 348: aaa2151.

Ritsma L, Ellenbroek SI, Zomer A, Snippert HJ, de Sauvage FJ, Simons BD, Clevers H, van Rheenen J. 2014. Intestinal crypt homeostasis revealed at single-stem-cell level by in vivo live imaging. Nature 507: 362-365.

Roesch K, Jadhav AP, Trimarchi JM, Stadler MB, Roska B, Sun BB, Cepko CL. 2008. The transcriptome of retinal Muller glial cells. J Comp Neurol 509: 225-238.

Saez B, Ferraro F, Yusuf RZ, Cook CM, Yu VW, Pardo-Saganta A, Sykes SM, Palchaudhuri R, Schajnovitz A, Lotinun S, et al. 2014. Inhibiting stromal cell heparan sulfate synthesis improves stem cell mobilization and enables engraftment without cytotoxic conditioning. Blood 124: 2937-2947.

Shook B, Rivera Gonzalez G, Ebmeier S, Grisotti G, Zwick R, Horsley V. 2016. The role of adipocytes in tissue regeneration and stem cell niches. Annu Rev Cell Dev Biol 32: 609-631.

Spinella-Jaegle S, Rawadi G, Kawai S, Gallea S, Faucheu C, Mollat P, Courtois B, Bergaud B, Ramez V, Blanchet AM, et al. 2001. Sonic hedgehog increases the commitment of pluripotent mesenchymal cells into the osteoblastic lineage and abolishes adipocytic differentiation. J Cell Sci 114: 2085-2094.

Srinivas S, Watanabe T, Lin CS, William CM, Tanabe Y, Jessell TM, Costantini F. 2001. Cre reporter strains produced by targeted insertion of EYFP and ECFP into the ROSA26 locus. BMC Dev Biol 1: 4.

St-Jacques B, Dassule HR, Karavanova I, Botchkarev VA, Li J, Danielian PS, McMahon JA, Lewis PM, Paus R, McMahon AP. 1998. Sonic hedgehog signaling is essential for hair development. Curr Biol 8: 1058-1068.

Suh JM, Gao X, McKay J, McKay R, Salo Z, Graff JM. 2006. Hedgehog signaling plays a conserved role in inhibiting fat formation. Cell Metab 3: 25-34.

Tamplin OJ, Durand EM, Carr LA, Childs SJ, Hagedorn EJ, Li P, Yzaguirre AD, Speck NA, Zon LI. 2015. Hematopoietic stem cell arrival triggers dynamic remodeling of the perivascular niche. Cell 160: 241-252.

Tontonoz P, Spiegelman BM. 2008. Fat and beyond: the diverse biology of PPAR $\gamma$. Annu Rev Biochem 77: 289-312.

Uezumi A, Fukada S, Yamamoto N, Takeda S, Tsuchida K. 2010. Mesenchymal progenitors distinct from satellite cells contribute to ectopic fat cell formation in skeletal muscle. Nat Cell Biol 12: 143-152. 
Woo WM, Zhen HH, Oro AE. 2012. Shh maintains dermal papilla identity and hair morphogenesis via a Noggin-Shh regulatory loop. Genes Dev 26: 1235-1246.

Xin T, Greco V, Myung P. 2016. Hardwiring stem cell communication through tissue structure. Cell 164: 1212-1225.

Youssef KK, Lapouge G, Bouvree K, Rorive S, Brohee S, Appelstein O, Larsimont JC, Sukumaran V, Van de Sande B, Pucci D, et al. 2012. Adult interfollicular tumour-initiating cells are reprogrammed into an embryonic hair follicle progenitor-like fate during basal cell carcinoma initiation. Nat Cell Biol 14: 1282-1294.

Yue R, Zhou BO, Shimada IS, Zhao Z, Morrison SJ. 2016. Leptin receptor promotes adipogenesis and reduces osteogenesis by regulating mesenchymal stromal cells in adult bone marrow. Cell Stem Cell 18: 782-796.

Zhang LJ, Guerrero-Juarez CF, Hata T, Bapat SP, Ramos R, Plikus MV, Gallo RL. 2015. Innate immunity. Dermal adipocytes protect against invasive Staphylococcus aureus skin infection. Science 347: 67-71.

Zhou M, Leiberman J, Xu J, Lavker RM. 2006. A hierarchy of proliferative cells exists in mouse lens epithelium: implications for lens maintenance. Invest Ophthalmol Vis Sci 47: 2997-3003.

Zhou BO, Ding L, Morrison SJ. 2015. Hematopoietic stem and progenitor cells regulate the regeneration of their niche by secreting Angiopoietin-1. Elife 4: e05521. 


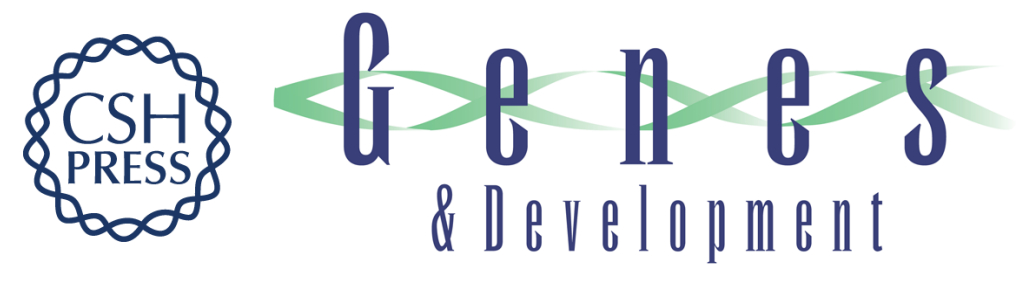

\section{Hair follicles' transit-amplifying cells govern concurrent dermal adipocyte production through Sonic Hedgehog}

Bing Zhang, Pai-Chi Tsai, Meryem Gonzalez-Celeiro, et al.

Genes Dev. 2016, 30: originally published online November 2, 2016

Access the most recent version at doi:10.1101/gad.285429.116

\section{Supplemental http://genesdev.cshlp.org/content/suppl/2016/11/02/gad.285429.116.DC1 \\ Material}

References This article cites 66 articles, 13 of which can be accessed free at:

http://genesdev.cshlp.org/content/30/20/2325.full.html\#ref-list-1

Creative This article is distributed exclusively by Cold Spring Harbor Laboratory Press for the first Commons six months after the full-issue publication date (see

License http://genesdev.cshlp.org/site/misc/terms.xhtml). After six months, it is available under a Creative Commons License (Attribution-NonCommercial 4.0 International), as described at http://creativecommons.org/licenses/by-nc/4.0/.

Email Alerting Receive free email alerts when new articles cite this article - sign up in the box at the top Service right corner of the article or click here.

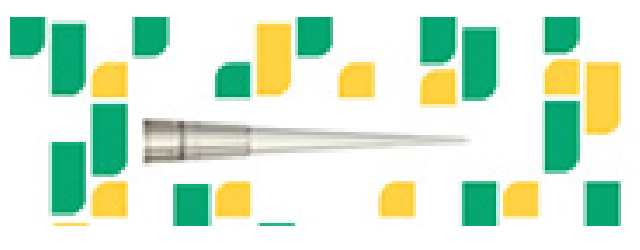

Focused on your science. 